\title{
Dynamique de la résorption utérine chez la chèvre du Sahel : effet de la parité
}

\author{
Boureima TRAORE ${ }^{1 *}$, Moussa ZONGO ${ }^{1}$, Were PITALA ${ }^{2}$, Moise HARO ${ }^{1}$, \\ Drissa SANOU $^{1}$ et Laya SAWADOGO ${ }^{1}$ \\ ${ }^{1}$ Laboratoire de Physiologie Animale, UFR/SVT, Université Ouaga I. Pr Joseph KI-ZERBO, \\ 03 BP 7021 Ouagadougou 03, Burkina Faso. \\ ${ }^{2}$ Ecole Supérieure d'Agronomie, Université de Lomé, BP 1515 Lomé, Togo. \\ *Auteur de correspondant ; E-mail : traoreboureima43@yahoo.fr; Tél : (00226) 75055167.
}

\section{REMERCIEMENTS}

Ce travail a été rendu possible grâce au soutien financier, matériel et technique du Programme de Productivité Agricole en Afrique de l'Ouest (PPAAO), FCN/2014-03, l'Agence Internationale de l'Energie Atomique (AIEA) et de la Fondation Internationale pour la Science. Les auteurs adressent leurs sincères remerciements à ces trois institutions.

\section{RESUME}

L'involution utérine est un facteur limitant pour la maitrise de la reproduction après la parturition chez les chèvres. L'objectif de cette étude est d'évaluer l'effet de la parité sur la dynamique de la résorption utérine par échographie chez dix-neuf $(n=19)$ chèvres du sahel réparties en deux groupes dont 11 primipares et 8 pluripares. Les examens ont été pratiqués par voie transrectale au moyen d'une sonde linéaire de $5 \mathrm{MHz}$ à intervalle réguliers. Les durées moyennes de la résorption utérine complète sont de 21,87 $\pm 2,93$ jours pour les primipares et de $22,75 \pm 2,31$ jours pour les pluripares. Ces délais n'ont pas été significativement différents entre les deux groupes de femelles $(\mathrm{p}=0,12)$. La résorption des caroncules utérines a été complète au $15^{\mathrm{e}}$ jour dans les deux groupes $(p=0,29)$. Le profil de la résorption utérine suit trois phases de régression. Une forte régression a été observée au cours de la première semaine $(7,23 \mathrm{~mm} /$ jour et $6,54 \mathrm{~mm} / \mathrm{jour})$. La vitesse de résorption a été moyenne $(1,13$ et $2,23 \mathrm{~mm} / \mathrm{jour})$ au cours de la deuxième et troisième semaine, et faible $(0,25$ et $0,23 \mathrm{~mm} / \mathrm{jour}$ ) à la quatrième semaine dans les deux groupes $(\mathrm{p}=0,018)$. Les résorptions utérines chez les primipares et les pluripares suivent une régression de type logarithmique $\left(\mathrm{R}_{1}{ }^{2}=0,98\right.$ et $\left.\mathrm{R}_{2}{ }^{2}=0,98\right)$ dont les équations respectives sont de $Y_{2}=-24,85 \ln (x)+100,85$ et $Y_{1}=-21,34 \ln (x)+88,271$. Chez la chèvre du Sahel en élevage contrôlé, la parité ne semble pas influencer la durée de la résorption utérine.

(c) 2017 International Formulae Group. All rights reserved.

Mots clés : Chèvre, résorption utérine, parité, échographie, parturition. 


\title{
Dynamics of uterine resumption in sahelian goats: effect of parity
}

\begin{abstract}
Uterine involution is a limited factor for the reproduction control after parturition in goats. The objective of this study is to evaluate the effect of parity on uterine resumption dynamic after parturition in nineteen $(n=19)$ Sahelian goats shared in two groups (primiparous $(n=11)$, pluriparous $(n=8))$. Ultrasound examinations were carried out every two days transrectally by using a real time, B-mode ultrasound scanner with a linear-array transducer of 5.0 MHz. The means durations of complete uterine resumption were $21.87 \pm$ 2.93 days in primiparous and $22.75 \pm 2.31$ days in pluriparous. However, times of complete uterine resumption in primiparous and pluriparous were not significantly different $(p=0.12)$. The resumption of uterine carunculars occurred completely at day 15 after parturition in all goats of the two groups $(\mathrm{p}=0,29)$. Uterine resumption in Sahelian goats indicates three phases of regression in the two groups, acute regression (7.23 $\mathrm{mm} /$ day and $6.54 \mathrm{~mm} /$ day $)$ in first week, moderate regression phase $(1.13$ and $2.23 \mathrm{~mm} /$ day $)$ in second and third week, and low regression $(0.25$ and $0.21 \mathrm{~mm} / \mathrm{day})$ at fourth week. The uterine resumptions followed logarithmic regression modals $\left(\mathrm{R} 12=0,97\right.$ and $\left.\mathrm{R} 2^{2}=0,98\right)$ which respective equations are: $\mathrm{Y} 2=-24.85 \ln (\mathrm{x})+$ 100.85 and $\mathrm{Y} 1=-21.34 \ln (\mathrm{x})+88.271$ respectively in pluriparous and primiparous. In monitoring breed, parity does not affect time of uterine resumption after parturition in Sahelian goat.
\end{abstract}

(C) 2017 International Formulae Group. All rights reserved.

Keywords: Goat, uterine resumption, parity, ultrasound, parturition.

\section{INTRODUCTION}

Une meilleure connaissance de la dynamique de l'utérus au cours du postpartum est importante pour une optimisation des productions animales (Ababneh et Degefa, 2005). La durée de l'intervalle entre les mises bas successives est dépendante du processus de résorption utérine et de la reprise de l'activité ovarienne cyclique après la parturition (Greyling, 2000 ; Kandiel et al., 2012). La durée moyenne de la résorption utérine complète chez les chèvres est comprise dans l'intervalle [16 - 28 jours] (Sanchez et al., 2002; Degefa et al., 2006 ; Badawi et al., 2014) et chez les brebis entre [17 - 40 jours] (Hauser et Bostedt, 2002; Gray et al., 2003). La résorption utérine est principalement influencée par la parité (Hajurka et al., 2005; Zhang et al., 2010), la race (Gray et al., 2003; Zduńczyk et al., 2004), l'allaitement et la nutrition (Greyling, 2000). Cependant, les effets de la parité sur les délais de la résorption utérine complète sont controversés chez les ruminants domestiques et méconnus chez la chèvre. Chez les brebis et les vaches, les primipares ont présenté une durée d'involution utérine plus longue que les pluripares (Zduńczyk et al., 2004, Zhang et al., 2010). Toutefois, des observations contraires ont été rapportées chez les vaches par Hajurka et al. (2005) et les bufflesses par Presicce et al. (2005).

Plusieurs techniques d'évaluation (hormonales, laparotomie, radiographie et échographie) de la résorption utérine sont utilisées chez les petits ruminants (Hussain et al., 2016). Aujourd'hui, l'échographie transrectale est appliquée en routine chez la chèvre pour contrôler l'involution utérine et la dynamique folliculaire grâce à son efficacité, sa fiabilité et sa praticabilité (Kandiel et al., 2012 ; Badawi et al., 2014, Zongo et al., 2015).

La chèvre du sahel Burkinabè est une chèvre locale d'Afrique de l'Ouest qui s'adapte bien au climat sahélien. En plus, elle a une activité sexuelle non saisonnière et un cycle de reproduction court (Traoré et al., 
2006 ; Zongo et Meyer, 2009). La fonction de reproduction de la chèvre du sahel au cours du post-partum reste peu connue et constitue un handicap majeur à la promotion de la race. Zongo et al. (2015) ont suivi l'involution utérine et la reprise de l'activité ovarienne par échographie chez la chèvre du sahel. Les résultats obtenus ont montré que l'activité ovarienne reprend une semaine après la parturition et que l'involution utérine est complète trois à quatre semaine postpartum. Dans l'optique d'une maîtrise de la reproduction au cours du postpartum, la présente étude a pour objectif d'apprécier par échographie l'effet de la parité sur la dynamique de la résorption utérine complète après la parturition chez la chèvre du sahel en condition d'élevage contrôlé.

\section{MATÉRIEL ET MÉTHODES}

\section{Animaux et traitements}

Cette étude a été réalisée à la station expérimentale de l'Université Ouaga I professeur Joseph Ki Zerbo, située à Gampéla $\left(12^{\circ} 22^{\prime}\right.$ latitude Nord et $1^{\circ} 31^{\prime}$ longitude Ouest) au Burkina Faso. Elle a concerné 19 chèvres du sahel en postpartum d'âge moyen $28,5 \pm 7,6$ mois et de poids moyens $32,5 \pm 7,3$ Kilogrammes. Cet effectif était constitué de 11 femelles primipares et 8 femelles pluripares (toutes $\geq 2$ ). Les animaux ont tous été préalablement contrôlés et déclarés cliniquement indemnes de toute infection parasitaire ou génitale. Ils n'ont pas non plus présenté d'antécédents pathologiques majeurs des fonctions de reproduction et de la lactation avant leur mise en expérimentation. Ils ont ensuite été maintenus dans des conditions standard et uniformes de conduite de l'élevage de la station expérimentale de Gampéla. Le troupeau de l'expérience a été gardé en stabulation libre. Leur alimentation a été essentiellement constituée de fourrage vert de niébé ou d'arachide additionné avec 1 kilogramme par animal et par jour de concentrés alimentaires (tourteau). Les chèvres ont été induites en œstrus et saillies par deux boucs fertiles. Elles ont été diagnostiquées gestantes un mois plus tard par échographie transrectale (Chison medical Imagin Co. Ldt, 8300, 5 Mhz linear transducer).

\section{Examens échographiques}

Les examens échographiques ont porté sur les femelles ayant connu une gestation et une mise bas normales dont les 19 femelles suivies. Ils ont été réalisés à intervalles deux jours à partir de $\mathrm{J} 1$ postpartum au moyen d'un échographe de type Chison Ultrasonic Scanner (Chison medical imagin co. 1td, 8300) équipé d'une sonde linéaire de 5-MHz (Badawi et al., 2014). Le câble de la sonde a été rigidifié avec un tube en plexiglass afin de faciliter l'introduction de la sonde et sa guidance dans le rectum.

L'animal, au cours de l'examen, est maintenu en station debout dans un couloir de contention. La sonde est enduite de gel et introduite délicatement dans le rectum de la chèvre afin d'éviter les blessures. La vessie est le repère, une fois observée, l'opérateur avance juste la sonde afin de mieux voir les deux cornes utérines. L'image de la section transversale du diamètre de la corne utérine est gelée à l'écran et mesurée. Sur chaque animal, trois à quatre mesures sont prises au cours de chaque examen afin de calculer les moyennes. La présence et le diamètre des caroncules utérines sont enregistrés au cours de chaque examen. 


\section{Analyse statistique}

Les critères d'une résorption utérine complète sont l'absence de variation significative entre les dimensions de trois dernières mesures successives (Badawi et al., 2014), la symétrie des deux cornes utérines avec un diamètre transversale inférieur ou égal à $2 \mathrm{~cm}$ et l'absence totale de lochies dans la cavité utérine (Zduńczyk et al., 2004).

Le diamètre moyen des caroncules utérines a été mesuré pour apprécier leur délai de résorption. Les données ont été exprimées sous forme de moyennes \pm écart type et soumises au test pair de Student (t-test paired) du logiciel R version 3.3 .2 (2016-10-31) de Foundation of Statistical Computing. Les différences sont considérées comme statistiquement significatives au seuil de $\mathrm{P} \leq$ 0.05 .

\section{RESULTATS}

La durée moyenne de la gestation chez la chèvre sahélienne a été de 145,93 $\pm 5,68$ jours avec des valeurs extrêmes comprises entre [144 - 153] jours. Les mesures échographiques moyennes de diamètres utérins et des caroncules utérines chez les chèvres primipares et pluripares sont présentées dans le Tableau 1. Les diamètres utérins ont été significativement plus grands chez les pluripares que les primipares ( $\mathrm{P}=$ 0,003). Cependant, les diamètres caronculaires n'ont pas varié significativement entre les deux groupes $(\mathrm{p}=0,29)$.

De J1PP à J23PP le diamètre utérin a diminué $(<20 \mathrm{~mm})$ considérablement pour les deux groupes (Tableau 1). A cette date, la résorption utérine a été complète chez $72,72 \%$ des femelles primipares et chez $62,50 \%$ des femelles pluripares.
La durée moyenne de résorption utérine chez la chèvre du Sahel est de 22,31 $\pm 3,02$ jours comprise entre 19 et 27 jours. Elle a été de 21,87 $\pm 2,93$ jours chez les chèvres primipares et de $22,75 \pm 2,31$ jours chez les chèvres pluripares. La différence de variation des délais moyens de l'involution utérine complète chez les femelles primipares et les femelles pluripares n'a pas été significative ( $p$ $=0,12$ ).

Les vitesses de réduction des diamètres utérins ont été calculées en fonction de la durée post-partum pour chaque parité et enregistrées dans le Tableau 2. L'analyse comparative des vitesses de résorption indique une différence significative entre les deux groupes $(p=0,018)$. Ces résultats obtenus dans les deux groupes montrent trois phases de régression (forte, moyenne et lente) du diamètre de la corne utérine. Une phase de forte régression $(p<0,01)$ a été observée au cours de la première semaine $(7,23 \mathrm{~mm} /$ jour et $6,54 \mathrm{~mm} /$ jour). La vitesse de résorption baisse considérablement et devient moyenne au cours de la deuxième et troisième semaine post-partum. Après la troisième semaine la vitesse de résorption devient très faible $(0,25$ et $0,21 \mathrm{~mm} /$ jour) dans les deux groupes. A $\mathrm{J} 23 \mathrm{PP}$, les taux de réduction du diamètre utérin ont été de $79,52 \%$ et de 79,55\% respectivement chez les chèvres primipares et pluripares.

Une charte de résorption utérine a été établie pour chaque parité par un nuage de points ajuster d'une courbe de tendance logarithmique. Les équations de régression obtenues exprimant la mesure du diamètre en fonction des jours a été de $\mathrm{Y}_{2}=-24,85 \ln (\mathrm{x})+$ 100,85 chez les femelles primipares. Chez les chèvres pluripares la variation du diamètre de la corne utérine en fonction du jour postpartum est de $\mathrm{Y}_{1}=-21,34 \ln (\mathrm{x})+88,271$. 
Les coefficients de détermination pour les primipares et les pluripares ont été respectivement $R^{2}=0,98$ et $R^{2}=0,98$ (Figure $1)$.

L'évaluation échographique de la résorption des caroncules indiquent qu'au cours des cinq premiers jours post-partum, les caroncules utérines sont de grandes tailles $(17,49 \pm 3,18$ et $14,77 \pm 5,29 \mathrm{~mm})$ respectivement chez les primipares et les multipares. A cette date, la cavité utérine est remplie de lochies (Figure 2 J1PP et Figure 3). A J7PP, elles sont réduites au maximum, soit $10,48 \pm 2,01$ pour les primipares et 10,27 \pm $3,21 \mathrm{~mm}$ pour les multipares (Figure $2 \mathrm{~J} 7 \mathrm{PP}$ ). $\mathrm{Au}$ quinzième jour après la mise bas, les caroncules utérines et des fluides utérins sont totalement résorbées (Figure2 J15PP, J23PP).

Tableau 1: Variations des diamètres utérins et caronculaires après la mise bas chez la chèvre du sahel.

\begin{tabular}{ccccc}
\hline \multirow{2}{*}{$\begin{array}{c}\text { Jours postpartum } \\
(\mathbf{m m})\end{array}$} & \multicolumn{2}{c}{ Diamètre corne utérine $(\mathbf{m m})$} & \multicolumn{2}{c}{ Diamètre caroncule utérine $(\mathbf{m m})$} \\
\cline { 2 - 5 } & Primipares & Pluripares & Primipares & Pluripares \\
\hline 1 & $92,00 \pm 5,33$ & $96,00 \pm 8,65$ & $17,49 \pm 3,18$ & $14,77 \pm 5,29$ \\
3 & $67,13 \pm 13,45$ & $81,29 \pm 4,48$ & $15,89 \pm 2,23$ & $12,14 \pm 2,11$ \\
5 & $51,71 \pm 6,25$ & $67,77 \pm 8,83$ & $14,99 \pm 5,79$ & $11,465 \pm 1,08$ \\
7 & $41,37 \pm 9,52$ & $50,24 \pm 1,16$ & $10,48 \pm 2,01$ & $10,27 \pm 3,21$ \\
9 & $36,35 \pm 6,69$ & $40,66 \pm 5,25$ & $9,19 \pm 0,72$ & $9,01 \pm 1,77$ \\
11 & $36,60 \pm 6,54$ & $41,20 \pm 11,61$ & $7,44 \pm 0,97$ & $5,43 \pm 0,56$ \\
13 & $32,52 \pm 6,16$ & $35,74 \pm 1,12$ & & \\
15 & $32,18 \pm 4,68$ & $33,12 \pm 9,97$ & & \\
17 & $29,47 \pm 1,52$ & $30,82 \pm 1,74$ & & \\
19 & $26,83 \pm 0,85$ & $27,18 \pm 1,74$ & & \\
21 & $23,26 \pm 1,33$ & $23,55 \pm 1,02$ & & \\
23 & $18,85 \pm 1,23$ & $19,64 \pm 0,85$ & & \\
25 & $17,78 \pm 0,52$ & $18,79 \pm 0,74$ & & \\
27 & $17,17 \pm 0,38$ & $18,75 \pm 1,49$ & & \\
29 & $18,01 \pm 0,75$ & $18,50 \pm 2,00$ & & \\
31 & $17,90 \pm 0,28$ & $18,08 \pm 1,32$ & & \\
33 & $17,66 \pm 0,69$ & $17,29 \pm 1,11$ & & \\
35 & $17,65 \pm 1,20$ & $17,30 \pm 1,01$ & & \\
\hline
\end{tabular}

Tableau 2 : vitesse de résorption du diamètre utérin par semaine et selon la parité.

\begin{tabular}{lcc}
\hline $\begin{array}{l}\text { Durée post-partum } \\
\text { (semaine) }\end{array}$ & \multicolumn{2}{c}{ Vitesse de résorption $(\mathbf{m m} / \mathbf{j o u r})$} \\
\cline { 2 - 3 } & Primipares & Pluripares \\
\hline 1 & 7,23 & 6,54 \\
2 & 1,13 & 2,23 \\
3 & 1,05 & 2,00 \\
4 & 0,21 & 0,25 \\
\hline
\end{tabular}




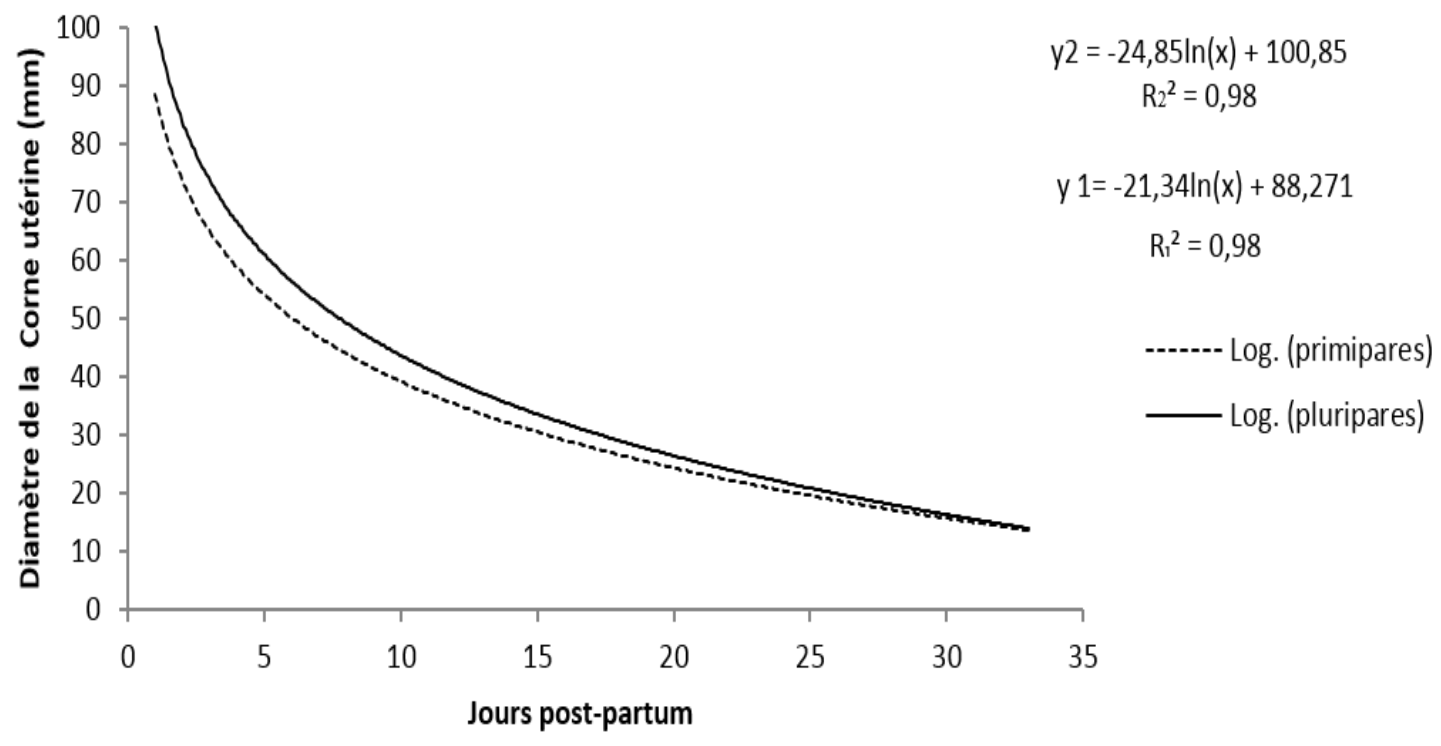

Figure 1 : Courbes de variation de l'involution utérine au cours du post-partum chez les femelles.

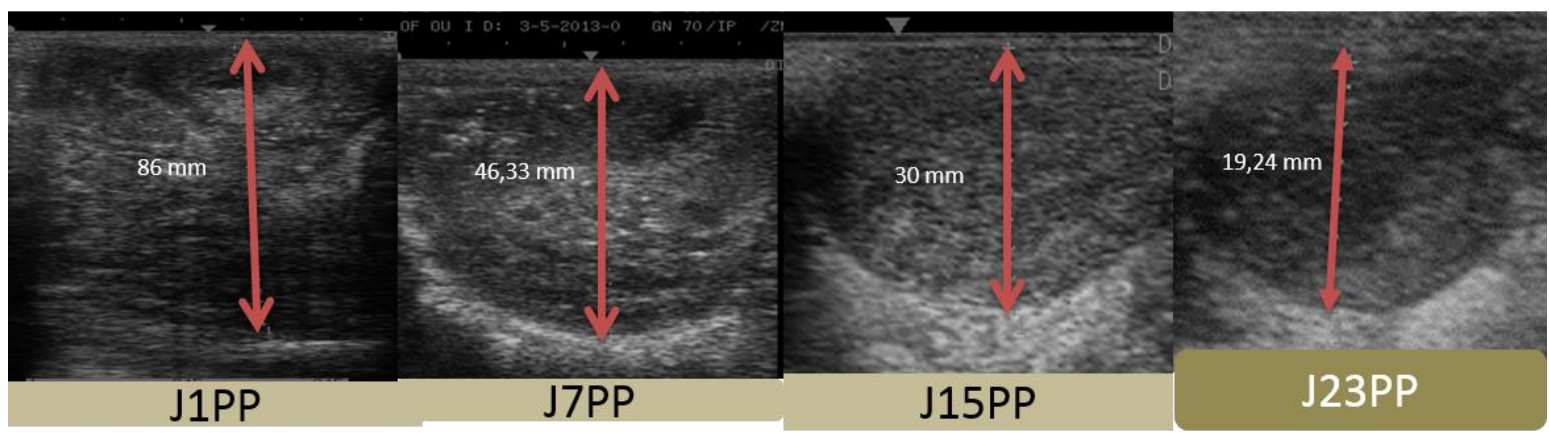

FIGURE 2 : Images échographiques de la corne utérine à J1, J7, J15 et J23 post-partum (JPP : jour post-partum).

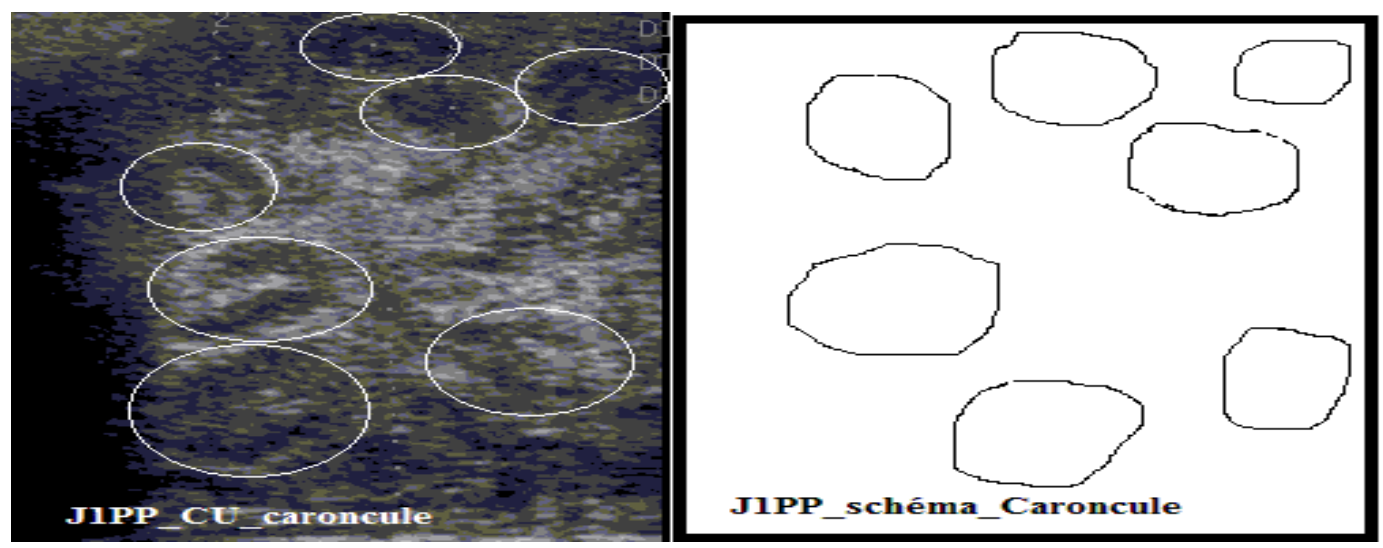

Figure 3 : Image échographique de la corne utérine portant des caroncules utérines. 


\section{DISCUSSION}

La durée moyenne de la gestation de la chèvre du sahel dans la présente étude se situe dans le même intervalle de temps rapporté par Mbaïndingatoloum (2011) en Afrique centrale sur la même race. La chèvre du sahel conserve ses principaux paramètres de reproduction quel que soit le milieu agro-écologique.

La durée moyenne de la résorption utérine complète chez les chèvres du sahel dans les conditions de cette expérience a été de 22,31 $\pm 3,02$ jours. Cette observation corrobore bien avec les données rapportées par Zongo et al. (2015) sur la même race en conditions d'élevage contrôlées. Des données comparables ont en outre été rapportées chez différentes autres races caprines d'Europe par Hauser et Bosted (2002), Ababneh et Degefa (2005), et Badawi et al. (2014). Ces valeurs sont cependant légèrement au-dessus de 30 jours et plus rapportées par Takayama et al. (2010). Zdunczyk et al. (2004), et Hayder et Ali (2008) chez la brebis. Cette variabilité des intervalles moyens de la résorption utérine seraient probablement liées aux conditions d'élevages, aux races, à l'allaitement et d'autres facteurs environnementaux (saison de mise bas, alimentation). L'alimentation et l'allaitement interférent avec l'activité de l'axe hypothalamo-hypophysaire-ovarien entraînant une suppression de la libération pulsatile de $\mathrm{LH}$ et la maturation finale des follicules dominants (Monniaux et al., 2009).

Le délai de la résorption utérine complète sont plus allongés chez les femelles pluripares $(22,75 \pm 2,31$ jours $)$ que chez les femelles primipares $(21,87 \pm 2,93$ jours $)$ avec une différence non significative $(\mathrm{p}=0,12)$. Ces observations traduiraient une faible influence de la parité sur la résorption utérine chez la chèvre du sahel comme précédemment rapportés chez les brebis à laine de la Pologne par Zduńczyk et al. (2004) et chez les vaches laitières de race Gir par Hajurka et al. (2005). A l'opposé, Degefa et al. (2006) et Hayder et Ali (2008) ont rapporté que le délai de la résorption utérine augmente avec celle de la parité.
L'effet et le mécanisme d'action de la parité sur la reprise de la cyclicité restent encore imprécis et controversés. Zhang et al. (2010), et Tanaka et al. (2008) expliquent que le mécanisme d'action de la parité est fonction de la balance énergétique de l'animal.

La résorption utérine chez la chèvre du Sahel a été caractérisée par 3 phases de régression et un taux de réduction du diamètre de la corne utérine. Des observations similaires ont été rapportées par Hayder et Ali (2008) et Hauser et Bostedt (2002) chez les brebis. En outre, Ababneh et Degefa (2005), Degefa et al. (2006) ont rapporté un taux de réduction de plus de $80 \%$ au cours de la première semaine post-partum. Cette phase de réduction rapide est suivie de la résorption complète des caroncules utérines et de la disparition des lochies à J15PP. Ces observations seraient dues au taux élevé de la prostaglandine juste les jours suivant la mise bas (Badawi et al., 2014). L'évolution du taux de réduction du diamètre utérin serait contrôlée par l'augmentation progressive du taux de progestérone au cours du postpartum. Aucun effet significatif de la parité n'a été observé sur le taux de réduction du diamètre de la corne et des caroncules utérines après la mise bas.

Le profil de la résorption utérine chez la chèvre du sahel suit une régression du type logarithmique $\left(\mathrm{R}^{2}=0,98\right)$. Chez la brebis, l'équation de la courbe de régression est de type exponentiel (Hauser et Bostedt, 2002). Les courbes de régression de l'utérus chez les pluripares et les primipares ont la même allure et sont presque superposées. Les différences de variation du diamètre utérin entre les deux groupes ont été significatives au cours du postpartum $(\mathrm{p}=0,003)$. Cette variation est bien visible par les positions relatives des deux courbes et s'expliquerait par un élargissement du diamètre de la corne utérine avec l'augmentation de la parité.

\section{Conclusion}

Cette étude a permis d'apprécier l'effet de la parité sur la résorption utérine au cours 
du post-partum chez les chèvres du sahel. Les résultats montrent que le délai moyen de la résorption utérine, le taux réduction $\mathrm{du}$ diamètre de la corne utérine et des caroncules utérines au cours du post-partum varient très peu en fonction de la parité chez la chèvre sahel en condition d'élevage contrôlé. Ainsi, la dynamique de la résorption utérine ne varie pas en fonction de la parité chez la chèvre du sahel. Ces résultats montrent que les protocoles de conduite des chèvres du sahel après la mise bas pourraient intégrer ensemble les femelles primipares et pluripares.

\section{CONFLIT D'INTERETS}

Les auteurs déclarent qu'il n'y a aucun conflit d'intérêts.

\section{CONTRIBUTIONS DES AUTEURS}

BT est l'investigateur principal des travaux, MZ a encadré, suivi et orienté les travaux ; WP a participé à la lecture du manuscrit, MH a participé à la collecte des données, DS a coordonné l'unité de recherche, LS, a coordonné le laboratoire.

\section{REFERENCES}

Ababneh MM, Degefa TJ. 2005. Ultrasonic assessment of puerperal uterine involution in Balady goats. J. Vet. Med. A: Physiol. Pathol. Clin. Med., 52: 244428. DOI: $\quad 10.1111 / \mathrm{j} .1439$ 0442.2005.00718.x

Badawi ME, Makawi SEA, Abdelghafar RM, Ibrahim MT. 2014. Assessment of postpartum uterine involution and progesterone profile in Nubian goats (Capra hircus). J. Adv. Vet. Anim. Res., 1(2): 36-41. DOI: 10.5455/javar. 2014.a10.

Degefa T, Ababneh M, Moustafa M. 2006. Uterine involution in the post-partum Balady goat. Vet. Arhiv., 76: 119 -133. http://www-staro.vef.unizg.hr/vetarhiv/ papers/2006-76-2-3.pdf.

Gray CA, Stewart MD, Johnson GA, Spencer TE. 2003. Postpartum uterine involution in sheep: histoarchitecture and changes in endometrial gene expression. Reproduction, 125: 185-198. DOI: 10.1530/rep.0.1250185.

Greyling JPC. 2000. Reproduction traits in the Boar goat doe. Small Rumin. Res., 36: 171-177. DOI: http://dx.doi.org/ 10.1016/S0921-4488(99)00161-3.

Hajurka J, Macak V, Hura V. 2005. Influence of health status of reproductive organs on uterine involution in dairy cows. Bull. Vet. Inst. Pulawy, 49:53-58. http://www.piwet.pulawy.pl/jvetres/imag es/stories/pdf/20051/20051053058.pdf

Hauser B, Bostedt H. 2002. Ultrasonographic observations of the uterine regression in the ewe under different obstetrical conditions. J. Vet. Med. A: Physiol. Pathol. Clin. Med., 49(10):511-516. DOI: $\quad 10.1046 / j .1439-0442.2002$. 00496.x.

Hayder M, Ali A. 2008. Factors affecting the postpartum uterine involution and luteal function of sheep in the subtropics. Small Rum. Res., 79: 174-178. http://doi.org/10.1016/j.smallrumres.200 8.07.023.

Hussain SO, Hussain KA, Al-Ani AA. 2016. Study on post-partum uterine involution by Ultrasonography and progesterone profile in local goats in Iraq. The Iraqi Journal of Veterinary Medicine, 40(1): 151-156.

Kandiel MM, Watanabe G, Abou El Roos ME, Abdel-Ghaffar AE, Sosa GA, ElAzab A el-S, Nagaoka K, Li JY, Manabe N, Taya K. 2012. Follicular turnover and hormonal association in postpartum goats during early and late lactation. $J$. Reprod. Dev., 58(1): 61-68. http://doi.org/10.1262/jrd.11-012S.

Mbaïndingatoloum FM. 2011. Paramètres de reproduction et application de l'insémination artificielle pour l'amélioration de la productivité chez la chèvre sahélienne au Tchad. Thèse de Doctorat Unique, Université Polytechnique de Bobo-Dioulasso (Burkina Faso), 146p. 
Monniaux D, Caraty A, Clément F, Dalbièstran R, Dupont J, Fabre S, Gérard N, Mermillod P, Monget P, Uzbekova S. 2009. Développement folliculaire ovarien et ovulation chez les mammifères. Inra Prod. Anim., 22(2), 59-76. https://hal.inria.fr/hal-00793623

Presicce GA, Bella A, Terzano GP, De Santisc G, Senatore ME. 2005. Postpartum ovarian follicular dynamics in primiparous and pluriparous Mediterranean Italian buffaloes (Bubalus bubalis). Theriogenology, 63: 14301439 . http://doi.org/10.1016/ j.theriogenology.2004.07.003.

Sanchez MA, Garcia P, Menendez S, Sanchez B, Gonzalez M, Flores JM. 2002. Fibroblastic growth factor receptor (FGF-R) expression during uterine involution in goat. Anim. Reprod. Sci. 69: 25-35. http://doi.org/10.1016/S03784320(01)00169-5.

Takayama H, Tanaka T, Kamomae H. 2010. Postpartum ovarian activity and uterine involution in non-seasonal Shiba goats, with or without nursing. Small Rumin. Research, 88:

62-66. http://doi.org/10.1016/j.smallrumres.200 9.11.001.

Tanaka T, Arai M, Ohtani S, Uemura S, Kuroiwa T, Kim S, Kamomae H. 2008. Influence of parity on follicular dynamics and resumption of ovarian cycle in postpartum dairy cows. Anim. Reprod. Sci., 108: 134-143. http://doi.org/10.1016/j.anireprosci.2007. 07.013 .
Traoré A, Tamboura HH, Kaboré A, Yaméogo N, Bayala B, Zaré I. 2006. Caractérisation morphologique des petits ruminants (ovins et caprins) de race locale "Mossi" au Burkina Faso. Anim. Genetic Resources Information, 39: 3950. DOI: https://doi.org/10.1017/ S1014233900002121

Zduńczyk S, Milewski S, Barański W, Janowski T, Szczepański W, Jurczak A., RAŚ A, Leśnik M. 2004. Postpartum uterine involution in primiparous and pluriparous polish longwool sheep monitored by ultrasonography. Bull. Vet. Inst. Pulawy, 48: 255-257. http://jvetres.piwet.pulawy.pl/images/sto ries/pdf/20043/20043255258.pdf

Zhang J, Deng LX, Zhang HL, Hua GH, Han L, Zhu Y, Meng XJ, Yang LG. 2010. Effects of parity on uterine involution and resumption of ovarian activities in postpartum Chinese Holstein dairy cows. J. Dairy Sci., 93: 1979 - 1986. DOI: http://dx.doi.org/10.3168/jds.2009-2626.

Zongo M, Traoré B, Ababneh MM, Hanzen C. Sawadogo L. 2015. Ultrasonographic assessment of uterine involution and ovarian activity in West Africa Sahelian goats. Journal of Veterinary Medicine and Animal Health, 7(2): 71-76. DOI: 10.5897/JVMAH2014.0322.

Zongo M, Meyer C, 2009. Variation saisonnière de la reproduction des ovins. Synthèse. Annales de l'Université de Ouagadougou, Série C., 7: 72. 